\title{
Study on drug resistance of Pseudomonas aeruginosa plasmid-mediated AmpC $\beta$-lactamase
}

\author{
BIN ZHU ${ }^{1}$, PING ZHANG ${ }^{1}$, ZHEN HUANG $^{1}$, HAI-QING YAN ${ }^{1}$, \\ AN-HUA WU ${ }^{2}$, GANG-WEN ZHANG ${ }^{1}$ and QIANGUO MAO ${ }^{3}$ \\ ${ }^{1}$ Department of Infectious Disease, The First Affiliated Hospital of Xinxiang Medical University, \\ Xinxiang 453100; ${ }^{2}$ Center of Infectious Control, Xiangya Hospital Center South University, Xiangya 410008; \\ ${ }^{3}$ Department of Hepatology, Xiamen Traditional Chinese Medicine Hospital, Xiamen 361004, P.R. China
}

Received July 25, 2012; Accepted November 9, 2012

DOI: $10.3892 / \mathrm{mmr} .2012 .1235$

\begin{abstract}
The aim of the present study was to investigate the resistance of plasmid-mediated AmpC $\beta$-lactamase in Pseudomonas aeruginosa, to detect and identify the AmpC genotype and to provide evidence for antibiotic applications in the clinic. Resistance phenotype in 108 strains of clinically isolated $P$. aeruginosa was determined by Kirby-Bauer disk test and cefoxitin three dimensional test in AmpC-positive strains. Plasmids were extracted from AmpC-positive strains using the SDS-alkali splitting technique. The depurated plasmid was used to amplify AmpC $\beta$-lactamase genes by PCR. Positive PCR products were sequenced by the Shanghai Sangon Biological Engineering Technology Company. Gene homology of PCR products with other index sample gene sequences was compared. In the present study, $28 \mathrm{AmpC}$ enzyme-positive $P$. aeruginosa strains among 108 were identified. Multidrug-resistance to antibiotics was observed in positive AmpC P. aeruginosa strains and a new P. aeruginosa strain of plasmid-mediated CMY-7 type AmpC enzyme was identified. In addition, AmpC type $\beta$-lactamases were revealed to be important in the resistance mechanism to antibiotics in P. aeruginosa. This is the first report of CMY-7 plasmid-mediated AmpC enzyme expression in P.aeruginosa.
\end{abstract}

\section{Introduction}

The increasing use and application of broad-spectrum antibiotics has led to increased reports of bacterial drug resistance and clinical infection. At present, drug-resistant bacteria, including methicillin-resistant Staphylococcus aureus, multidrug-resistant Streptococcus pneumoniae, vancomycinresistant Enterococcus, multidrug-resistant Baumanii,

Correspondence to: Professor Bin Zhu, Department of Infectious Disease, The First Affiliated Hospital of Xinxiang Medical University, Jinshuida Road, Xinxiang 453100, P.R. China

E-mail: zbahcn@163.com

Key words: Pseudomonas aeruginosa, plasmid, ampC enzyme resistance
Klebsiella pneumoniae, Escherichia coli and Pseudomonas aeruginosa are increasingly being detected in clinical and laboratory settings (1). Appearance of pan-resistant bacteria, including Cray Borrelia, which produces New Delhi Metallo $\beta$-lactamase-1, an enzyme responsible for generating resistance (2), is causing an even greater challenge to humans. Therefore, study of clinical bacterial drug resistance and mechanisms and the development of solutions to bacterial resistance is important. Previously, Gram-negative bacilli AmpC $\beta$-lactamase was understood to be generated by chromosome mediation only. However, Papanicolaou et al (3) identified plasmid-mediated AmpC $\beta$-lactamase (MIR-1) in a strain of Cray Borrelia bacterium isolated from a patient in Rhode Island Hospital (USA) in 1989. Following this, the plasmid-mediated AmpC enzyme was rapidly identified in a number of other countries. At present, more than 20 plasmid-mediated AmpC enzymes have been identified. The plasmid-mediated AmpC enzyme is resistant to antibiotics, enabling the plasmid to continue to express the protein at high levels and carry multiple drug-resistant genes. Plasmid-mediated drug resistance is transmitted between members of the same or different bacterial species. Transmission is rapid and performed at a range of distances. Therefore, understanding of the mechanism and elucidation of solutions to plasmid-mediated AmpC enzyme resistance is crucial.

$P$.aeruginosa is one of the main bacterial strains associated with hospital infections, including burns, cystic pulmonary fibrosis and ventilator-associated infections. In addition, $P$. aeruginosa is resistant to multiple antibiotics and is associated with high rates of mortality (4-6). A number of drug resistance mechanisms have been identified in P. aeruginosa, including production of $\beta$-lactamase (extended-spectrum $\beta$-lactamases or AmpC) (7), alteration of penicillin-binding protein expression (8), formation of biomembranes, expression of cytomembrane permeability barriers $(9,10)$ or active multidrug efflux pumps, including mex-ABoprM (11) and alterations in channel protein expression, including oprD $(11,12)$. At present, $P$. aeruginosa plasmid-mediated AmpC $\beta$-lactamase has not been studied. The present study aimed to investigate plasmid-mediated AmpC in clinically-isolated P. aeruginosa through identification of the genetype of a drug-resistant strain, to aid clinical antibiotic applications. 


\section{Materials and methods}

Specimens. In the present study, 108 strains of $P$. aeruginosa were clinically isolated from the sputum, urine, feces, ascites, hydrothorax, arthroedema, cerebrospinal fluid and other specimens of hospitalized patients in the ICU, respiratory, cardiology, infection, burns and pediatrics departments in the First Affiliated Hospital of Xin Xiang Medical University and Xiang Ya Hospital Center South University. Bacterial isolation, culture and identification was conducted according to the National Guide to Clinical Laboratory Procedures (2nd edition) and bacterial identification was conducted using an automatic bacterial identification analyzer. E. coli ATCC 25922 and $P$. aeruginosa ATCC27853 were used as quality-control strains and Aerobacter cloacae $029 \mathrm{M}$ was the used as a positive control strain as it is known to express the AmpC enzyme. The study was evaluated and approved by the ethics committees of Xin Xiang Medical University (XinXiang, China) and Xiang-Ya Medical College of Central South University (Changsha, China). Informed patient consent was obtained from the patient or the patient's family.

Primary screening test of specimens. Isolated $P$. aeruginosa was inoculated onto a blood plate and cultured at $37^{\circ} \mathrm{C}$ overnight. The following day, cultures were suspended in $0.9 \%$ stroke-physiological saline solution (Maxwell turbidity, 0.5) and coated uniformly onto $\mathrm{M}-\mathrm{H}$ plates with sterile cotton swabs. Cefoxitin (FOX), ceftazidime (CAZ), cefotaxime/clavulanic acid (CTX/CA) and imipenem (IMP) drug-sensitive slips were symmetrically placed onto the $\mathrm{M}-\mathrm{H}$ plates with bacterial suspension using sterile forceps and cultured overnight at $37^{\circ} \mathrm{C}$. The next day, bacteriostatic rings of drug-sensitive slips were observed and the diameters of bacteriostatic rings were measured with a vernier caliper. According to criteria prepared by the National Clinical Laboratory Standardization Committee of America (13), bacteriostatic ring diameters of $\leq 18, \leq 21, \leq 22$ and $\geq 13 \mathrm{~mm}$ for FOX, CTX/CA, CAZ and IMP, respectively, were indicative of strains expressing the AmpC enzyme.

Preparation of AmpC enzyme. P. aeruginosa stains identified by screening to express the AmpC enzyme were inoculated into $10 \mathrm{ml} \mathrm{LB}$ broth and cultured overnight in a thermostat shaker at a speed of $80-100 \mathrm{rpm}$ at $37^{\circ} \mathrm{C}$. Subsequently, the culture mixture was centrifuged in a refrigerated centrifuge at $4^{\circ} \mathrm{C}$ to remove the supernatant. Bacterial pellets were frozen for $30 \mathrm{~min}$ at $-80^{\circ} \mathrm{C}$ and then thawed in a water bath. The freezethaw cycle was performed 5 times. Following this, bacteria were suspended in $1.5 \mathrm{ml}$ PBS buffer $(0.01 \mathrm{M})$ in an EP tube, mixed uniformly by agitation and centrifuged at low temperature to obtain the supernatant. The supernatant contained the AmpC enzyme and was filtered using a $0.22-\mu \mathrm{m}$ microporous membrane. Next, the filtrate was inoculated onto the blood plate and cultured overnight at $37^{\circ} \mathrm{C}$. Bacterial growth was observed and negative filtrates were stored at $-20^{\circ} \mathrm{C}$.

Three-dimensional test. E. coli strain ATCC25922 was suspended in solution (Maxwell turbidity, 0.5) and uniformly coated with sterile cotton swabs onto M-H plates. A FOX drug-sensitive slip $(30 \mu \mathrm{g})$ was applied to the center of the plate and a sterile surgical blade was used to cut a small groove with a width of $\sim 1-2 \mathrm{~cm}, 3 \mathrm{~mm}$ away from the slip edge. In each small groove, 10-20 $\mu \mathrm{l}$ AmpC enzyme extract of each test bacterium was added, avoiding extract overflow. The extract was cultured overnight at $37^{\circ} \mathrm{C}$ for $18-24 \mathrm{~h}$ prior to observation. The Bacillus levans $(0.29 \mathrm{M})$ strain was used as a positive control as it is known to produce the AmpC enzyme. If the cefoxitin three-dimensional test shows a positive result, it suggests that the test bacterium generated the AmpC enzyme.

Plasmid extraction and electrophoresis. P. aeruginosa plasmids with positive three-dimensional test results were extracted by the SDS alkaline lysis method and then run on a $1 \%$ agarose gel. Electrophoresis was performed using $0.5 \mathrm{X}$ TBE electrophoresis buffer and images were captured under UV lamp.

PCR amplification. Plasmid-mediated AmpC $\beta$-lactamasepositive strains were divided into 6 populations according to chromosome source: i) ACC (Hafinia alvei); ii) FOX (unknown source); iii) MOX (unknown source); iv) DHA (Morganella morganii); v) CIT (Citrobacter freundii); or vi) EBC population (A. cloacae). Primer design was performed using AmpC enzyme gene sequences identified in previous reports and primer 5.0 software (Table I).

PCR system. Total PCR volume was $50 \mu 1$ and contained the following: $5 \mu \mathrm{l} 10 \mathrm{X}$ PCR buffer solution, $1 \mu \mathrm{ldNTP}$ $(10 \mathrm{mmol} / \mathrm{l}), 2 \mu \mathrm{l}$ positive and reverse primers $(100 \mathrm{pmol} / \mu \mathrm{l})$, $4 \mu 1 \mathrm{MgCl}_{2}$ (25 mmol/l), $0.4 \mu \mathrm{l} \mathrm{Taq}$ enzyme (2 units), $4 \mu \mathrm{l}$ template and $31.6 \mu 1 \mathrm{ddH}_{2} \mathrm{O}$. For each run, 6 reactions were performed. With the exception of primers, all other reaction components and volumes were constant. PCR conditions were as follows: initial denaturation for $3 \mathrm{~min}$ at $94^{\circ} \mathrm{C} ; 30$ cycles of denaturation for $30 \mathrm{sec}$ at $94^{\circ} \mathrm{C}$, annealing for $30 \mathrm{sec}$ at $58^{\circ} \mathrm{C}$, extension for $30 \mathrm{sec}$ at $72^{\circ} \mathrm{C}$; and a final extension for $2 \mathrm{~min}$ at $72^{\circ} \mathrm{C}$. Agarose gel electrophoresis of the PCR amplification products was performed and images were captured using a gel imaging instrument.

Sequencing and analysis. Sequencing of PCR amplification products was performed by the Shanghai Biological Engineering Technology and Services Co., Ltd. (Shanghai, China). PubMed BLAST sequence alignments were analyzed with DNAssist software.

\section{Results}

Three-dimensional test. Primary screening tests of K-B drug-sensitive slips of 108 strains of clinically-isolated $P$. aeruginosa demonstrated that 30 strains were AmpC enzyme-positive. Three-dimensional test of FOX confirmed that 28 strains were AmpC enzyme-positive.

Electrophoresis. The 28 positive strains of $P$. aeruginosa were analyzed by electrophoresis following plasmid extraction. Electrophoretic analysis revealed bands in 22 strains. Single and double bands were identified corresponding to $2 \mathrm{~kb}$ and/or $5 \mathrm{~kb}$. Single and double bands were observed in 7 and 15 strains, respectively (Fig. 1). 
Table I. AmpC enzyme primers.

\begin{tabular}{|c|c|c|c|c|}
\hline AmpC enzyme & Primer & Sequence $\left(5^{\prime}-3^{\prime}\right)$ & Length (bp) & Genbank no. \\
\hline MOX-1 MOX2 CMY-1 & MOX & GCT TGA GCG GTA AAC GAG TG & 171 & D 13304 \\
\hline CMY-8 CMY-11 & & GAA TGT GCT GCC TGG GTG & & \\
\hline LAT-1-LAT-4 & CIT & AGG GAT TAG GCT GGG AGA TG & 251 & X 78117 \\
\hline CMY-7 BIL-1 & & GAC ACG GAC AGG GTT AGG ATA G & & \\
\hline DHA-1 DHA-2 & DHA & $\begin{array}{l}\text { CCG TCT CCG TAA AGG GTA AGC } \\
\text { CGG TCA GAG CAC CAA ACA GG }\end{array}$ & 396 & Y 1641 \\
\hline ACC & $\mathrm{ACC}$ & $\begin{array}{l}\text { GTT ATC CGT GAT TAC CTG TC } \\
\text { TAC TCA GCG AAC CCA CTT }\end{array}$ & 248 & AJ 133121 \\
\hline MIR-1 ACT-1 & $\mathrm{EBC}$ & $\begin{array}{l}\text { GAA ATC CGC CAC ATC CTG } \\
\text { CCG TAA TAG CAG TTC AAG GGA }\end{array}$ & 462 & M 37839 \\
\hline FOX-1-FOX-5b & FOX & $\begin{array}{l}\text { CT ACA GTG CGG GTG GTT } \\
\text { CGG GCT TAT CTT CCT TCG }\end{array}$ & 297 & X 77455 \\
\hline
\end{tabular}

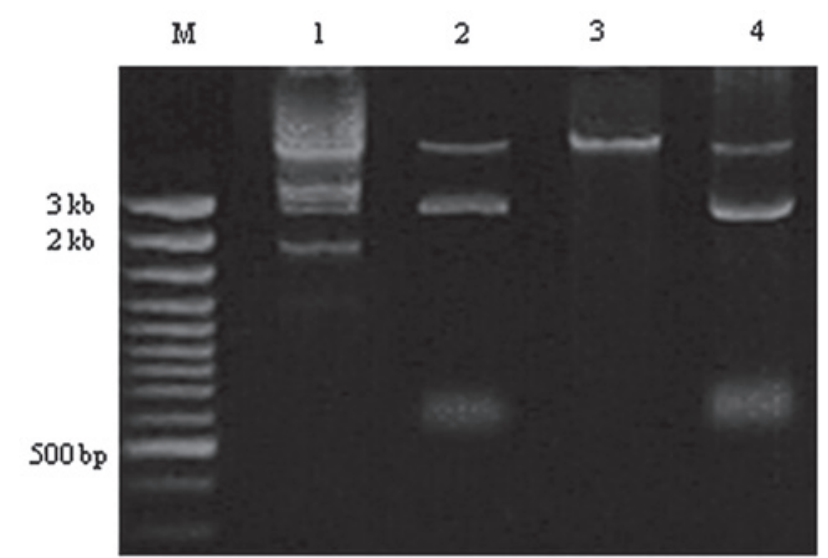

Figure 1. Plasmid DNA electrophoretogram. M, DNA marker; lane 1, Aerobacter cloacae positive control; lanes 2-4, Pseudomonas aeruginosa.

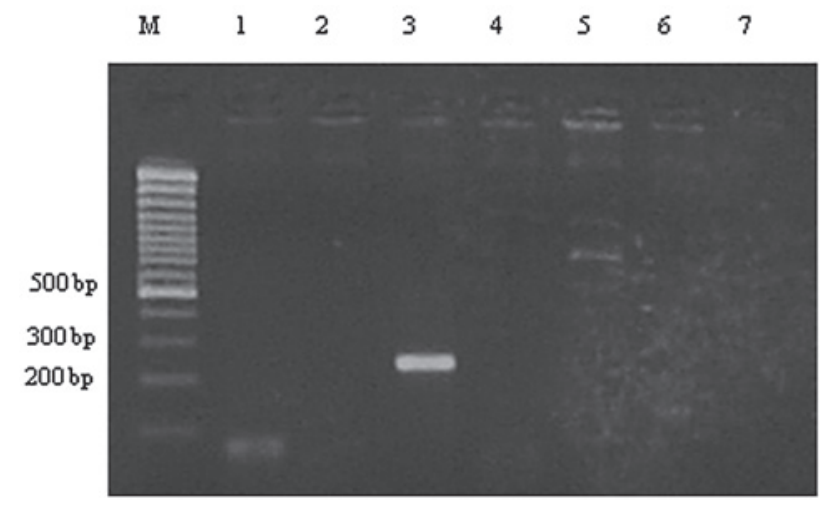

Figure 2. Electrophoretogram of PCR amplification products. M, DNA marker; lane 1, negative control; lane 3, positive product (251 bp); lanes 2 , 4-7, negative.

PCR amplification. Purified plasmid DNA was used for PCR amplification templates. For each DNA template, 6 reactions were performed. An amplification product was obtained using one pair of primers of a total of 6 primer pairs. This primer was of CIT type and the target length was $251 \mathrm{bp}$ (Fig. 2). Within 22 strains of plasmid-positive P. aeruginosa, a single purified PCR amplification product was positive for this band.

Sequencing analysis. The positive strain revealing a target band of $251 \mathrm{bp}$ was sequenced. The sequencing results are shown in Fig. 3.

Homology comparison. The sequenced product (2-220 bp) was identified to exhibit $99.5 \%$ (218/219) homology to the A. cloacae K9973 $\beta$-lactamase AmpC gene (881-1099 bp) The total length of the $\beta$-lactamase AmpC gene sequence in A. cloacae is 1165 bp (Fig. 4).

\section{Discussion}

$P$. aeruginosa is one of the main bacterial species responsible for severe hospital infections (14) and is resistant to a number of antibacterial drugs. $P$. aeruginosa has several drug-resistant mechanisms: 1) the generatation of $\beta$-lactamase enzymes; 2) efflux pumps (e.g. mex2oprM); 3) target-site or outer membrane modifications. Multiple drug resistance is usually the result of the chromosomal and plasmid-encoded AmpC enzymes (15-17). Drug resistance cannot currently be detected by routine antibiotic sensitivity tests and the detection of the process is complex (18). At present, no universal test exists for the detection of AmpC $\beta$-lactamase; however, the three-dimensional test was previously reported to be the most suitable detection method available (19).

In the present study, 108 strains of $P$. aeruginosa were screened using K-B drug-sensitive slips. An AmpC enzyme-positive result was observed in 30 strains and the three-dimensional test of FOX was performed, identifying 28 strains as AmpC enzyme-positive. The detection coincidence rate of the two methods for AmpC enzyme-positive stains was $93.3 \%$.

The three-dimensional test identified 28 AmpC enzyme-positive strains of $P$. aeruginosa. Following this, electrophoresis of plasmid extracts identified representative plasmid bands in 22 strains. Six strains were negative and had lost or did not carry plasmids initially. PCR amplification analysis identified positive plasmid-mediated AmpC enzyme expression in a 


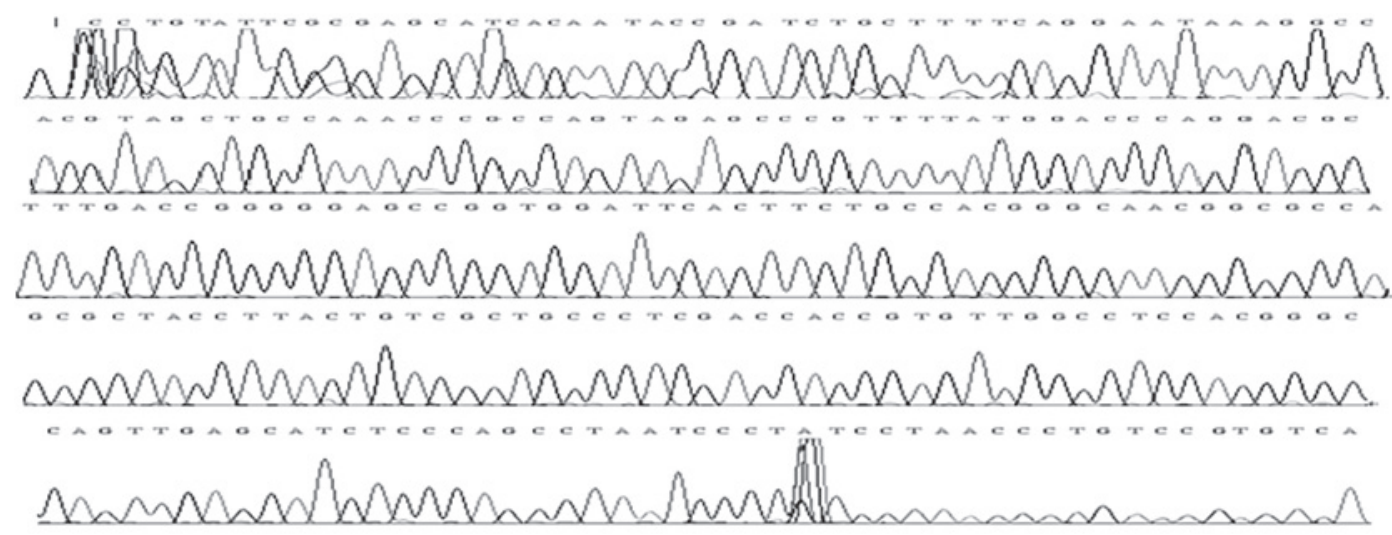

Figure 3. Sequencing of PCR products (251 bp).

\begin{abstract}
Query: 2 tgtattcgcgagcatcacaat accgatctgcttttcaggaataaaggccacgtagctgcc 61 |l|||||||||||||||||||||||||||||||||||||||||||||||||||||||||

Sbjct: 1099 tgtattcgcgagcatcacaat accgatctgcttttcaggaataaaggccacgtagct gcc 1040
\end{abstract}

\author{
Query: 62 aaacccgccagtagagcccgttttatggacccaggacgctttgaccgggggagccggtgg 121 \\ IIIIIIIIIIIIIIIIIIIIIIIIIIIIIIIIIIIIIIIIIIII \\ Sbjct : 1039 aaacccgccagtagagcccgttttatggacccaggacgctttgaccgggggagccggtgg 980
}

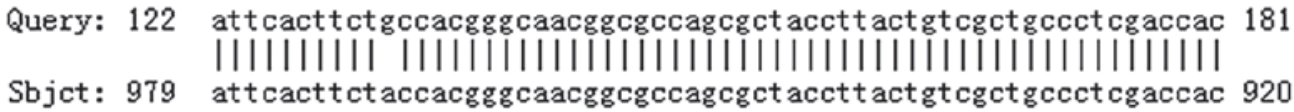

Query: 182 cgtgttggcctccacgggccagttgagcatctcccagcc 220 |11||1|||||||||||||||||||||||||||||||||||

Sbjct: 919 cgtgttggcctccacgggccagttgagcatctcccagcc 881

Figure 4. Sequence comparison of PubMed Bla.

single strain, demonstrating that the plasmid-mediated AmpC enzyme was present in $P$. aeruginosa; however, the proportion of plasmid-positive strains carrying the AmpC enzyme gene was low. It is possible that the $P$. aeruginosa strains that the three-dimensional test showed as positive could produce the majority of the chromosomal-mediated AmpC enzyme.

Previous studies on plasmid-mediated AmpC $\beta$-lactamase are largely restricted to the bacterila strains A. cloacae and Cray Borrelia (20-22). A limited number of studies have analyzed drug resistance in $P$. aeruginosa plasmid-mediated AmpC $\beta$-lactamase. In 2003, Shahid et al (23) isolated $P$. aeruginosa strains exhibiting plasmid-mediated AmpC enzyme from burns patients in an intensive care hospital in Northern India. In the domestic environment, a single strain of $E$. coli was previously observed to exhibit ACT-1 plasmid AmpC enzyme. However, no domestic reports on $P$. aeruginosa plasmid-mediated AmpC enzyme expression currently exist. The present study identified a single strain of $P$. aeruginosa plasmid-mediated AmpC enzyme expression using plasmid extraction, PCR amplification, product sequencing and BLAST homology comparison. A $99.5 \%$ homology with the A. cloacae K9973- $\beta$-lactamase AmpC gene sequence was identified. The overall length of A. cloacae K9973- $\beta$-lactamase AmpC gene sequence was $1,165 \mathrm{bp}$, consistent with the gene sequence of A. cloacae K9973-generating CMY-7 AmpC enzyme previously identified by Lee et al (24). The present study is the first reported identification of plasmid-mediated AmpC $\beta$-lactamase expression in P. aeruginosa.

\section{References}

1. Lister PD, Wolter DJ and Hanson ND: Antibacterial-resistant Pseudomonas aeruginosa: clinical impact and complex regulation of chromosomally encoded resistance mechanisms. Clin Microbiol Rev 22: 582-610, 2009.

2. Yong D, Toleman MA, Giske CG, et al: Characterization of a new metallo-lactamase gene, bla NDM-1 and a novel erythromycin esterase gene carried on a unique genetic structure in Klebsiella pneumoniae sequence type 14 from India. Antimicrob Agents Chemother 53: 5046-5054, 2009.

3. Papanicolaou GA, Medeiros AA and Jacoby GA: Novel plasmidmediated beta-lactamase (MIR-1) conferring resistance to oxyimino- and alpha-methoxy beta-lactams in clinical isolates of Klebsiella pneumoniae. Antimicrob Agents Chemother 34: 2200-2209, 1990

4. Driscoll JA, Brody SL and Kollef MH: The epidemiology, pathogenesis and treatment of Pseudomonas aeruginosa infections. Drugs 67: 351-368, 2007.

5. Obritsch MD, Fish DN, MacLaren R and Jung R: National surveillance of antimicrobial resistance in Pseudomonas aeruginosa isolates obtained from intensive care unit patients from 1993 to 2002. Antimicrob Agents Chemother 48: 4606-4610, 2004. 
6. Zhanel GG, DeCobry M, Adam H, et al: Prevalence of antimicrobial-resistant pathogens in Canadian hospitals: results of the Canadian Ward Surveillance Study (CANWARD 2008). Antimicrob Agents Chemother 54: 4684-4693, 2010.

7. Weldhagen GF, Poirel L and Nordmann P: Ambler class A extended-spectrum beta-lactamases in Pseudomonas aeruginosa: novel developments and clinical impact. Antimicrob Agents Chemother 47: 2385-2392, 2003.

8. Moyá B, Zamorano L, Juan C, Ge Y and Oliver A: Affinity of the new cephalosporin CXA-101 to penicillin-binding proteins of Pseudomonas aeruginosa. Antimicrob Agents Chemother 54: 3933-3937, 2010

9. Burmolle M, Thomsen TR, Fazli M, et al: Biofilmsin chronic infections - a matter of opportunity - monospecies biofilms in multispecies infections. FEMS Immunol Med Microbiol 59: 324-336, 2010

10. del Pozo JL and Patel R: The challenge of treating biofilm-associated bacterial infections. Clin Pharmacol Ther 82: 204-209, 2007.

11. Pfeifer Y, Cullik A and Witte W: Resistance to cephalosporins and carbapenems in gram-negative bacterial pathogens. Int J Med Microbiol 300: 371-379, 2010.

12. Nikaido H: Outer membrane barrier as a mechanism of antimicrobial resistance. Antimicrob Agents Chemother 33: 1831-1836, 1989.

13. Wikler MA, Cockerill, FR, Craig WA, et al: Methods for Dilution Antimicrobial Susceptibility Tests for Bacteria that Grow Aerobically. 7th edition. Clinical and Laboratory Standards Institute, Wayne, PA, 2006.

14. Vincent JL: Nosocomial infections in adult intensive care units Lancet 361: 2068-2077, 2003.

15. Carmeli Y, Troillet N, Eliopoulos GM and Samore MS Emergence of antibiotic-resistant Pseudomonas aeruginosa: comparison of risk associated with different antipseudomonal agents. Antimicrob Agents Chemother 43: 1379-1382, 1999.
16. Cavallo JD, Fabre R, Leblanc F, Nicolas-Chanoine $\mathrm{MH}$ and Thabaut A: Antibiotic susceptibility and mechanisms of beta-lactam resistance in 1310 strains of Pseudomonas aeruginosa: a French multicentre study (1996). J Antimicrob Chemother 46: 133-136, 2000.

17. Livermore DM: Multiple mechanisms of antimicrobial resistance in Pseudomonas aeruginosa: our worst nightmare? Clin Infect Dis 34: 634-640, 2002.

18. Thomson KS: Controversies about extended-spectrum and AmpC beta-lactamases. Emerg Infect Dis 7: 333-336, 2001.

19. Tan TY, Ng LS, He J, Koh TH and Hsu LY: Evaluation of screening methods to detect plasmid mediated AmpC in Escherichia coli, Klebsiella pneumoniae and Proteus mirabilis. Antimicrob Agents Chemother 53: 146-149, 2009.

20. Abdalhamid B, Wickman PA and Hanson ND: Correlation of ampC induction with PBP binding in Enterobacter cloacae. In: 45th Intersci Conf Antimicrob Agents Chemother. Washington DC, C1-2211, 2005.

21. Kuga A, Okamoto R and Inoue M: AmpR gene mutations that greatly increase class $\mathrm{C}$ beta-lactamase activity in Enterobacter cloacae. Antimicrob Agents Chemother 44: 561-567, 2000.

22. Pfeifer Y, Cullik A and Witte W: Resistance to cephalosporins and carbapenems in Gram-negative bacterial pathogens. Int J Med Microbiol 300: 371-379, 2010

23. Shahid M, Malik A and Sheeba: Multidrug-resistant Pseudomonas aeruginosa strains harbouring R-plasmids and AmpC beta-lactamases isolated from hospitalised burn patients in a tertiary care hospital of North India. FEMS Microbiol Lett 228: 181-186, 2003.

24. Lee SH, Kim JY, Shin SH, et al: Dissemination of SHV-12 and characterization of new AmpC-type beta-lactamases genes among clinical isolates of Enterobacter Species in Korea. J Clin Microbiol 41: 2477-2482, 2003. 